\title{
III. Aussenwirtschaftspolitik
}

\section{(2) OpenEdition \\ 1 Journals}

Electronic version

URL: http://journals.openedition.org/sjep/1024

DOI: $10.4000 /$ sjep.1024

ISSN: 1663-9677

\section{Publisher}

Institut de hautes études internationales et du développement

\section{Printed version}

Date of publication: 1 janvier 1985

Number of pages: $71-81$

ISSN: $1660-5926$

\section{Electronic reference}

«III. Aussenwirtschaftspolitik», Schweizerisches Jahrbuch für Entwicklungspolitik [Online], 5 | 1985,

Online erschienen am: 16 Februar 2013, abgerufen am 08 September 2020. URL : http://

journals.openedition.org/sjep/1024 ; DOI : https://doi.org/10.4000/sjep.1024 


\section{AUSSENWIRTSCHAFTSPOLITIK}

\section{EXPORTRISIKOGARANTIE}

1984 lag das Defizit der Exportrisikogarantie ERG mit 237 Mio Franken leicht unter dem Rekorddefizit von 239 Mio Franken des Vorjahres. Auf den 1. April 1985 wurden die Gebühren zur Absicherung politischer Risiken erhöht und die Währungsabsicherung sistiert. Der Grossteil der Entschädigungszahlungen der ERG erfolgte wiederum für Transferschäden und im Rahmen von Schuldenkonsolidierungsvereinbarungen. Rund 75\% der ERG-Verpflichtungen konzentrierten sich 1984 auf Exporte in die Dritte Welt. Die Hilfswerke fordern vermehrte Berücksichtigung entwicklungspolitischer Grundsätze bei der Gewährung der ERG, Einsitz von Vertretern der Hilfswerke in die ERG-Kommission sowie eine verstärkte Transparenz der ERG-Geschäfte.

Die ERG wurde 1934 zur Krisenbekämpfung geschaffen. Sie ist eine Versicherung des Bundes für besondere, mit Ausfuhrgeschäften verbundene Risiken. Mit der ERG versichern die schweizerischen Exporteure ihre Lieferungen gegen politische, Fabrikations- und Währungsrisiken (letzteres wurde am 1. April 1985 sistiert). Wichtigste Garantiesummen stellen aber das Transferrisiko und Schuldenkonsolidierungen dar (1).

Das Prinzip der ERG ist langfristig die Eigenwirtschaftlichkeit. Anhaltende Defizite machten in letzter Zeit bedeutende Vorschüsse aus der Bundeskasse nötig.

Die ERG schliesst für 1984 mit 150 Mio Franken Einnahmen und 387 Mio Franken Ausgaben mit einem Defizit von 237 Mio Franken (Vorjahr 239 Mio Franken), das wiederum durch Vorschüsse des Bundes gedeckt wurde. Diese erhöhen sich damit auf insgesamt 599 Mio Franken. Die ERG-Rechnung stellt diesen Vorschüssen die Guthaben des ERG-Fonds aus Schuldenkonsolidierungen von 630 Mio Franken gegenüber.

Die Garantien erreichten 1984 einen Betrag von 5,2 Mia Franken (Vorjahr 8,4). Davon entfielen 4,8 Mia Franken auf Garantien politischer Risiken und 0,4 Mia Franken auf Währungsgarantien. Die Gesamtverpflichtung der ERG beträgt 15,8 Mia Franken.

Bei den Ausgaben sind die Transferschäden stark gestiegen auf 175 Mio Franken (Vorjahr 132 Mio Franken). Die Ausgaben für Schuldenkonsolidierungen, bei denen die ERG im Umfang ihrer Auszahlungen vom Schuldnerland anerkannte Forderungen übernimmt, betrugen 159 Mio Franken (Vorjahr 167 Mio Franken). Die Schadenauszahlungen aus Währungsrisiken sind von 90 Mio Franken 1983 auf 31 Mio Franken stark zurückgegangen. 
Wie in den vergangenen Jahren hat auch im Berichtsjahr die Verschuldungskrise zu vermehrten Zahlungsverzögerungen und langfristigen Stundungsvereinbarungen geführt. Die schwierige Zahlungsbilanzsituation zahlreicher Entwicklungsländer lässt vermuten, dass die ERG noch für längere Zeit mit Zahlungsverzögerungen zu rechnen hat.

Die Gesamtverpflichtung der ERG von 15,8 Mia Franken verteilt sich wie folgt: 12,5 Mia Fr. oder $78,7 \%$ der Garantien sind für Lieferungen an Entwicklungsländer (gemäss Definition der OECD, d.h. einschliesslich OPECund Schwellenländer) übernommen worden. Auf die 67 "einkommensschwachen Entwicklungsländer" entfielen 2,7 Mia Fr. oder 17,2\%. Davon entfiel mehr als die Hälfte auf Aegypten, Indien und Indonesien. Ein Engagement von mehr als 50 Mio Franken weisen ferner weitere 8 Länder aus: China, El Salvador, Honduras, Kenia, Mali, Pakistan, Sudan, Togo.

Zahlungen im Rahmen von Schuldenkonsolidierungen wurden geleistet aufgrund von Vereinbarungen mit den Ländern Türkei, Togo, Senegal, Elfenbeinküste, Sierra Leone, Sudan, Jugoslawien, Peru, Sambia, Ekuador. Insgesamt sind 36 Konsolidierungsabkommen mit 17 Ländern von einem Total von 629,8 Mio Fr. in Kraft.

Das BAWI veröffentlicht keine Angaben über die einzelnen gewährten ERGZusicherungen nach Auftrag oder Land.

\section{Revision der ERG-Verordnung}

Der Bundesrat hat im Berichtsjahr einer weiteren Revision der Verordnung über die ERG zugestimmt. Auf den 1. April 1985 erhöhten sich die Gebühren zur Absicherung politischer Risiken um 45\%. Gleichzeitig wurde die Währungsabsicherung sistiert. Mit diesen Massnahmen soll die Finanzlage des ERG-Fonds verbessert werden.

Als ungenügend erachten die Hilfswerke in einer Stellungnahme die Revision der ERG-Verordnung. Sie gehe in die richtige Richtung, sei jedoch einseitig, weil sie die Entwicklungspolitik nicht einplane.

\section{ERG und Entwicklungspolitik}

In einer Eingabe an das EVD fordert die Arbeitsgemeinschaft von Swissaid, Fastenopfer, Brot für Brüder und Helvetas neue Wege in der ERG. Entwicklungspolitische Kriterien sollen im gesetzlichen Auftrag und in der Praxis der ERG stärker berücksichtigt werden. Seit 1981 ist der Bund verpflichtet, bei Exporten in die ärmeren Entwicklungsländer seine Grundsätze der Entwicklungspolitik mitzuberücksichtigen. Diese Klausel führte jedoch noch nie zur Ablehnung eines Gesuchs. So wurden auch Anteile des umstrittenen Baus des Manantali-Staudamms in Mali durch die ERG versichert.

Weiter kritisieren die Hilfswerke die für die ERG geltende Liste der "ärmeren Entwicklungsländer". Diese zählt gemäss OECD die 67 Entwicklungsländer mit einem Pro-Kopf-Einkommen von unter 600 US-Dollar. Die Arbeitsge- 
meinschaft fordert, dass diese Staatengruppe gleich wie im Rahmen der wirtschafts- und handelspolitischen Massnahmen der Entwicklungszusammenarbeit (u.a. Mischkredite) die Länder mit Einkommen bis 1400 US-Dollar miteinbezieht.

In der Praxis der ERG sollen die Gesuche unter dem Gesichtspunkt geprüft werden, ob das betreffende Projekt letztlich die Eigenständigkeit der Wirtschaft in der Dritten Welt stärkt.

Nuklearmaterial, militärisch verwendbare Flugzeuge (PC-7) und generell Güter mit "fragwürdigem Nutzen für die breite Bevölkerung" sind von der ERG auszuschliessen.

Schliesslich verlangen die Hilfswerke und die Gewerkschaften ein Mitspracherecht in der einseitig aus Vertretern der Wirtschaft und der Verwaltung zusammengesetzten ERG-Kommission und eine bessere Transparenz.

Zur Sanierung der Finanzlage des ERG-Fonds schlugen die Hilfswerke u.a. Prämienerhöhungen für die Wirtschaft vor und eine Zurückhaltung der Garantiegewährung bei entwicklungspolitisch fragwürdigen Grossprojekten, wie das Atatürk-Kraftwerk in der Türkei. Eine Umwandlung der Bundesvorschüsse in eigentliche Zuschüsse lehnen sie ab.

\section{Anmerkung}

1) Wir verweisen auf die in diesem Jahrbuch im Teil "Untersuchungen und Stellungnahmen" ausführlich wiedergegebene Diskussion zwischen verschiedenen Interessenvertretern zu aktuellen Fragen der ERG.

Ein weiterer ausführlicher Diskussionsbeitrag ist die Studie von Markus Mugglin: “Exportrisikogarantie, Internationale Verschuldung und Schweizerische Entwicklungspolitik", verfasst im Auftrag der Arbeitsgemeinschaft und des Instituts für Sozialethik des SEK, Adliswil 1985.

Quellen

EVD-Pressemitteilung vom 22.5.1985 und vom 18.3.1985.

Arbeitsgemeinschaft Swissaid, Fastenopfer, Brot für Brüder und Helvetas: Neue Wege der ERG, Brief an das EVD vom 5.2.85.

$N Z Z$ 1.3.1985.

TA 19.3.1985.

\section{INTERNATIONALE WAEHRUNGSHILFE}

Das Parlament hat an seiner Frühjahrssession 1985 den Bundesbeschluss, welcher den Bundesrat ermächtigt, zur Verhütung oder Behebung ernsthafter Störungen der internationalen Währungsbeziehungen an internationalen Stüt- 
zungsaktionen zugunsten anderer Währungen teilzunehmen, um weitere zehn Jahre (ab 15.7.1985) verlängert. Der maximale Verpflichtungsrahmen wurde dabei von bisher 2 Mia auf 1 Mia Franken reduziert.

Die Schweiz spielt im Rahmen der internationalen Währungshilfe eine aufgrund ihrer stark überschüssigen Ertragsbilanz wichtige Rolle.

Mit Beschluss vom Dezember 1983 über den Beitritt der Schweiz zu den Allgemeinen Kreditvereinbarungen AKV wurde die internationale Währungshilfe auf eine Rechtsgrundlage gestellt. Der Bundesrat kann die Nationalbank mit der Durchführung der Operationen beauftragen.

Der maximale Verpflichtungsrahmen wurde auf die Hälfte herabgesetzt, weil die Schweiz als Mitglied der AKV ihre Aktionen hauptsächlich in diesem Rahmen abwickeln will. Zudem trägt die Halbierung des finanziellen Rahmens dem Umstand Rechnung, dass der Bund die Durchführung von Interventionen an der Währungsfront zumeist der Nationalbank überlässt und sich auf die Gewährung einer Garantie beschränkt.

Der mit einer Bundesgarantie versehene Kredit der Nationalbank an Portugal von 30 Mio US-Dollar wurde im September 1984 vollständig zurückbezahlt. Somit waren Ende 1984 noch zwei bilaterale Kredite mit Bundesgarantie ausstehend: Türkei mit 45,5 Mio US-Dollar und Jugoslawien mit 80 Mio USDollar.

Eine von der Arbeitsgemeinschaft Swissaid/Fastenopfer/Brot für Brüder und Helvetas verlangte entwick lungspolitisch ausgerichtete Zusatzbestimmung zum Bundesbeschluss wurde nicht berücksichtigt.

\section{Quellen}

Bundesbeschluss über die Mitwirkung der Schweiz an internationalen Währungsmassnahmen vom 20. März 1975.

Stellungnahme der Arbeitsgemeinschaft vom 12. März 1984.

Nationalbank, 77. Geschäftsbericht, Bern 1985.

NZZ 6.9.1984 und 15.3.1985.

TA 6.9.1984.

\section{SCHULDENKONSOLIDIERUNGSABKOMMEN}

Auf die multilateralen Umschuldungsverhandlungen im Rahmen des Pariser Klubs folgen die bilateralen Schuldenkonsolidierungsabkommen.

In der Berichtsperiode hat die Schweiz derartige Abkommen mit Kuba, Jugoslawien und mit sechs afrikanischen Entwicklungsländern in Höhe von insgesamt 207 Mio Fr. abgeschlossen. (Einzelheiten sind aus nachstehender Tabelle ersichtlich.) 


\begin{tabular}{lrc}
\hline & Datum & Mio Fr. \\
Jugoslawien & 26.7 .1984 & 60,0 \\
Elfenbeinküste & 31.8 .1984 & 80,0 \\
Togo IV & 4.9 .1984 & 30,0 \\
Madagaskar III & 14.9 .1984 & 3,7 \\
Sudan IV & 3.12 .1984 & 17,0 \\
Sambia II & 14.12 .1984 & 5,7 \\
Kuba II & 22.1 .1985 & 3,5 \\
Senegal IV & 11.6 .1985 & 7,1 \\
Total & & 207,0 \\
\hline
\end{tabular}

Quelle: BAWI

Weiter steht die Schweiz für Abkommen mit folgenden Ländern in Verhandlung: Peru (drittes Abkommen), Jamaika, die Philippinen, Argentinien, Ekuador (zweites Abkommen), Madagaskar (viertes Abkommen).

Mit anderen Ländern, die in der Berichtsperiode ihre Schulden im Rahmen des Pariser Klubs neu verhandelten, schliesst die Schweiz aus Effizienzgründen keine bilateralen Abkommen ab, da der Mindestbetrag der betroffenen Summe (1 Mio SZR) nicht erreicht wird. Es handelt sich um: Mosambik, Niger, Liberia, Somalia, Costa Rica, Mauretanien, die Dominikanische Republik und Zaire.

Quelle

BAWI

\section{ZOLLPRAEFERENZEN}

Aufgrund des Zollpräferenzbeschlusses kann ein grosser Teil der Waren aus den Entwicklungsländern zollfrei in die Schweiz eingeführt werden. Gewisse Produkte der Bereiche Landwirtschaft, Textilien, Bekleidung und Schuhe sind jedoch weiterhin zollpflichtig. Dafür werden aber nicht die normalen Zollansätze berechnet, sondern es gelten Präferenzzölle. Im Rahmen der siebten Zollabbaustufe des GATT hat der Bundesrat die Präferenz-Zollansätze zugunsten der Entwicklungsländer auf den 1. Januar 1985 neu angepasst. 


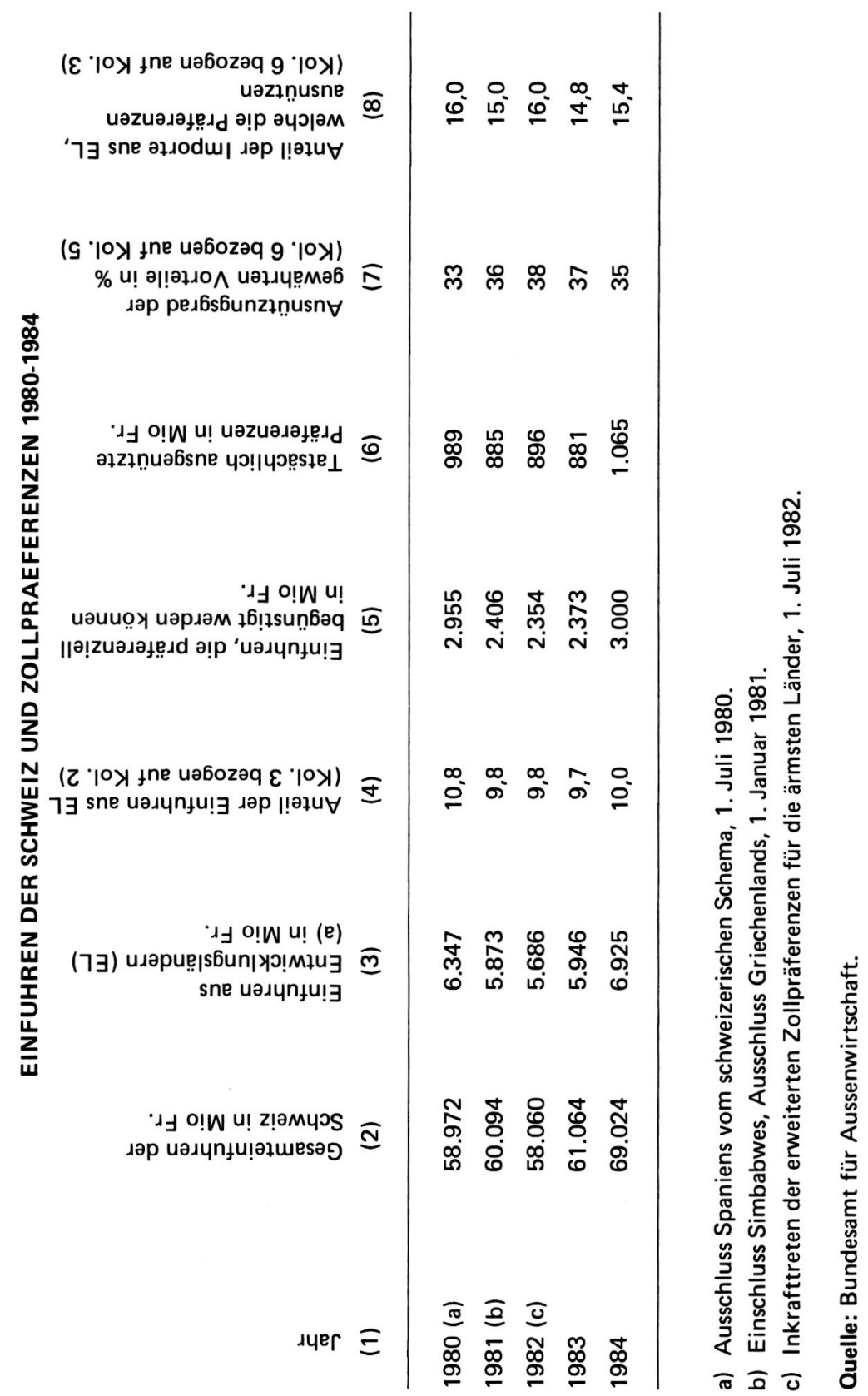


1984 importierte die Schweiz für 6,9 Mia Franken Waren aus Entwicklungsländern (Vorjahr 5,9 Mia) (1). Davon hätten Importe in der Höhe von $3 \mathrm{Mia}$ Franken präferenziell begünstigt werden können. Der Ausnutzungsgrad der gewährten Präferenzen betrug 35\%. Der schwache Ausnutzungsgrad für Präferenzzölle erklärt sich dadurch, dass für Edelsteine und Edelmetalle, die sehr niedrigen Zöllen unterliegen, ganz selten Zollpräferenzen verlangt werden.

Die Schweiz unterstützt mit finanziellen Mitteln die von der UNCTAD zur besseren Ausnutzung der Zollpräferenzen gewährte technische Hilfe an die Entwicklungsländer.

\section{Anmerkung}

1) In den Statistiken der schweizerischen Aussenwirtschaft zählt die Bundesverwaltung Jugoslawien und Israel zu den Entwicklungsländern, nicht aber die Türkei (Mitglied der OECD).

\section{WIRTSCHAFTSGESPRAECHE}

In der Berichtsperiode wurden zwischen der Schweiz und einzelnen Entwicklungsländern in verschiedenen Formen Wirtschaftsgespräche geführt. Wir erwähnen hier nur die wichtigsten.

Im September 1984 erörterte die gemischte Kommission Schweiz-Jugoslawien für wirtschaftliche, kommerzielle, industrielle und wissenschaftlichtechnische Zusammenarbeit die Entwicklungsperspektiven der bilateralen Handelsbeziehungen und prüfte Möglichkeiten der Intensivierung der gegenseitigen Beziehungen (1).

An der Internationalen Messe für Bauwesen und chemische Industrie von Teheran im September 1984 nahmen 66 Aussteller aus der Schweiz teil (2).

Im Oktober 1984 erörterten Bundesrat Aubert und eine Schweizer Delegation auf einer Reise durch die vier lateinamerikanischen Länder Argentinien, Venezuela, Kolumbien und Mexiko nebst politischen Fragen auch bilaterale Wirtschaftsprobleme. Die Frage der schweizerischen Investitionen in diesen Ländern, deren Verschuldung, sowie der Schutz von geistigem Eigentum für Marken und Patente standen dabei im Zentrum der Gespräche (3).

Im November 1984 unterzeichneten die Schweiz und Aegypten einen Vertrag über die Zusammenarbeit auf dem Gebiet der friedlichen Nutzung der Kernenergie. Der Vertrag, welcher noch ratifiziert werden muss, würde es der schweizerischen Exportindustrie ermöglichen, am umfangreichen nuklearen Ausbauprogramm in Aegypten (geplant sind 6 neue Kernkraftwerke bis ins Jahr 2005, im Umfang von 36 Mia US-Dollar) mitzumachen. Das Geschäft ist 
für die Wintersession 1985 im Parlament vorgesehen. Mit Botschaft vom 1. 1. Mai 1985 beantragt der Bundesrat beim Parlament die Benehmigung des Vertrages. Die Hilfswerke kritisieren an der Botschaft das Fehlen entwicklungspolitischer Ueberlegungen. Aegypten gehört mit 580 US-Dollar Pro-Kopf-Einkommen (1980) zu den ärmeren Entwicklungsländern, bei denen nach ERGGesetz die Grundsätze der schweizerischen Entwicklungspolitik mitzuberücksichtigen sind (mit Gesuchen der beteiligten schweizerischen Firmen an die ERG ist zu rechnen). Nach Ansicht der Hilfswerke scheint die nukleare Option Aegyptens im heutigen Zeitpunkt entwicklungspolitisch verfehlt, weshalb die Schweiz von jeglicher Beihilfe "zu dieser Fehlentwicklung über staatliche Förderungsmassnahmen absehen sollte" (4).

Die Schweiz führte in Dschidda/Saudiarabien erstmals eine schweizerische Industriemesse (Swissexpo 84 mit 130 schweizerischen Ausstellern) durch. Am Rande der Messe führten Bundesrat Furgler und eine Schweizer Delegation Gespräche über eine Verbesserung der wirtschaftlichen Zusammenarbeit zwischen der Schweiz und Saudiarabien. Es wurden insbesondere Fragen in Zusammenhang mit Joint-Venture-Verträgen erörtert. In Jordanien führte Bundesrat Furgler u.a. Gespräche über die Finanzierung von Projekten durch Mischkredite (5).

Im Januar 1985 fand in Kuala Lumpur/Malaysia und in Bangkok/Thailand das Wirtschaftssymposium Swiss Tech 85 statt. An diesen Veranstaltungen präsentierte eine Schweizer Delegation Technik und Know-how für den Export nach Südostasien. Aehnliche Swiss-Tech-Seminare waren bereits in Südkorea, auf Taiwan, in Indonesien und auf den Philippinen abgehalten worden (6).

Erstmals seit der Gründung Nordkoreas 1948 weilte eine offizielle Delegation aus Nordkorea im März 1985 zu Wirtschaftsgesprächen in der Schweiz (7).

Im Mai führte eine Schweizer Delegation Wirtschaftsgespräche in Südkorea, namentlich über eine Zusammenarbeit in den Wirtschaftsbereichen Textilien und Landwirtschaft. 1984 exportierte die Schweiz Waren im Wert von 133 Mio US-Dollar nach Südkorea, während sie für 82 Mio US-Dollar südkoreanische Produkte einführte (8).

In Mexiko fand im Mai 1985 die Tecno Suiza 85, eine Ausstellung von schweizerischen Unternehmen, statt. BAWI-Direktor Sommaruga führte mit Regierungsvertretern Gespräche über einen möglichen Ausbau der bilateralen Wirtschaftsbeziehungen (9).

\section{Quellen}

1) EVD-Pressemitteilung 3.9.1984.

2) NZZ 19.9.1984.

3) NZZ 18.10.1984.

4) NZZ 15.11.1984 und Dokumentation der Arbeitsgemeinschaft Swissaid/Fastenopfer/ Brot für Brüder/Helvetas zum "Zusammenarbeitsvertrag Schweiz-Aegypten auf dem Gebiet der Kernenergie", Bern 22.9.1985. 
5) EVD-Pressemitteilung 19.11.1984.

6) EVD-Pressemitteilung 15.1.1985.

7) Le Matin 9.3.1985.

8) EVD-Pressemitteilung 15.1.1985.

9) EVD-Pressemitteilung 9.5.1985.

\section{WAFFENAUSFUHR}

Die gesamten Kriegsmaterialexporte stiegen 1984 gegenüber dem Vorjahr um 15 Mio Fr. von 377 auf 392 Mio Fr. Damit scheint die rückläufige Entwicklung der Waffenausfuhr in den Jahren 1981-83 (Abnahme um 134 Mio Fr.) vorläufig zum Stillstand zu kommen. Die Bedeutung am Gesamtexport der Schweiz ist nach wie vor gering; sie betrug 1984 0,65\%.

Die Exporte nach Entwicklungsländern (1) stiegen um 50 Mio Fr. Gleichzeitig stieg auch der prozentuale Anteil von 28\% (1983) auf $40 \%$ der gesamten Kriegsmaterialexporte. Markant zugenommen haben innerhalb der europäischen Entwicklungsländer die Waffenausfuhren nach Griechenland, das mit 67 Mio Fr. den Hauptabnehmer aller Entwicklungsländer darstellt.

Bemerkenswerterweise figuriert Nigeria (1983 noch der wichtigste Kunde unter den Entwicklungsländern mit 59 Mio Fr.) nicht mehr unter den Abnehmern von schweizerischem Rüstungsmaterial. Diese Lücke wurde aufgefüllt durch die Golfstaaten Arabische Emirate (23 Mio), Saudiarabien (20 Mio) und Bahrain (18 Mio).

Die Exporte in die ärmeren Entwicklungsländer blieben praktisch konstant. 1984 wurde zum ersten Mal Kriegsmaterial nach Cina geliefert (0,6 Mio Franken). Siehe nachfolgende Tabelle.

\section{PC-7-Flugzeuge}

Um den Export von PC-7-Flugzeugen in Kriegsgebiete wie Guatemala und Iran wurde in der Junisession 1985 im Nationalrat eine heftige Debatte geführt. Der Export von Pilatus-PC-7-Trainings- und Aufklärungsflugzeugen untersteht gemäss Bundesratsbeschluss vom 11. März 1985 nicht dem Kriegsmaterialgesetz. Diese Leichtflugzeuge werden im Ausland (erwiesenermassen in Guatemala) zu Kampfflugzeugen umgebaut und als solche eingesetzt. Ein Postulat (Leuenberger, SP) forderte eine Aenderung der Verordnung zum Kriegsmaterialgesetz. In Zukunft sollten erfahrungsgemäss zu Kampfmitteln umgebaute Flugzeuge dem Kriegsmaterialgesetz unterstellt werden. Nach langer Debatte lehnte der Rat das Postulat ab.

Die Ausfuhr von PC-7-Flugzeugen kann durch die ERG abgesichert werden. Hilfswerke und Entwicklungsorganisationen hatten vom Bundesrat vergeblich einen Verzicht auf ERG-Deckung für den PC-7-Export gefordert. 


\section{Anmerkung}

1) Die hier angewandte Definition von Entwicklungsländern umschliesst sämtliche OPECLänder sowie Länder wie Spanien, Griechenland, Portugal, Türkei, Jugoslawien und Zypern (europäische Entwicklungsländer). Auch die asiatischen Staatshandelsländer (China, Mongolei, Vietnam, D.V.R. Korea) wurden in diese Kategorie aufgenommen.

\section{Quellen}

Institut für Sozialethik des SEK, Bern.

NZZ 29.1. und 21.6.1985.

TA 16.3.1985.

SCHWEIZERISCHE KRIEGSMATERIALEXPORTE 1983 UND 1984

(in $1000 \mathrm{Fr}$. und \%)

1. Gesamtexport

2. nach Indu strieländern

(ohne Staatshandelsländer)

3. nach Entwicklungsländern *

insgesamt

ohne OPEC

ohne OPEC und europäische

Entwicklungsländer

unterteilt nach Ländergruppen

\section{3}

$1000 \mathrm{Fr}$.

377.236

272,285

104.931

35.356

8.881

26.475

6.394

15.255

530

4.123

173

übrige (Jugoslawien, Zypern)

OPEC

Nigeria

Ekuador

Algerien

Arab. Emirate
69.575

59.310

5.641

1.687

2.879
1984

1000 Fr. $\quad \%$

$392.332 \quad 100$

\section{2}

234.522

60

$\begin{array}{lll}28 & 157.324 & 40\end{array}$

$\begin{array}{lll}9,5 & 103.629 & 26\end{array}$

$2,5 \quad 29.395$

7 
übrige (Saudiarabien, Katar, Kuwait,

Irak, Iran, Indonesien, Venezuela)

übrige ölexportierende

Entwicklungsländer

Bahrain

\author{
0
}

0

18.733

5

Entwicklungsländer der oberen

\title{
Einkommensgruppe (HEL)
}

2.032

1.976

1

1.985

1.895

übrige (Israel, Niederl. Antillen,

Hongkong, Argentinien)

56

0

90

0

Entwicklungsländer der mittleren

Einkommensgruppe (MEL)
Peru
Malaysia

$\begin{array}{rlrl}5.886 & 1,5 & 25.795 & 7 \\ 580 & 0 & 667 & 0 \\ 4.448 & 1,5 & 5.850 & 1,5 \\ 526 & 0 & 536 & 0\end{array}$

übrige (Chile, Uruguay, Tunesien, Angola, Libanon, Kolumbien, Süd-

korea, Taiwan, Costa Rica,

Dominik. Republik)

12

Entwicklungsländer der niedrigen

Einkommensgruppe (NEL)

- LLDC (Sudan, Botswana, Tansania)

963

- MSAC

9

Indien

360

übrige (Aegypten, Pakistan,

Elfenbeinküste, Kamerun, Kenia,

Senegal, Guyana, Burma, Madagaskar)

- übrige NEL

261

Marokko

Philippinen

Liberia

übrige (Jordanien, Thailand,

Swasiland)

117
594
574

sozialistische Entwicklungsländer

China

\begin{tabular}{rrrr}
117 & 0 & 640 & 0 \\
594 & 0 & 864 & 0 \\
574 & 0 & 761 & 0 \\
2 & 0 & 4 & 0 \\
0 & 0 & 4 & 0 \\
36 & 0 & 95 & 0 \\
& & & \\
& & 600 & 0 \\
\hline
\end{tabular}

* Abgrenzung der Entwicklungsländer und Gruppenbildungskriterien nach Höhn, Der Schweizerische Aussenhandel mit der Dritten Welt, Adliswil 1980, S. 19 ff. Sämtliche Absatzländer mit über $100000 \mathrm{Fr}$. Ausfuhrwert in mindestens einem Jahr werden einzeln aufgeführt, die übrigen zwar genannt, aber in der Sammelkategorie "übrige" zusammengefasst. 